\title{
Polysèmes
}

Revue d'études intertextuelles et intermédiales

$21 \mid 2019$

(Re)constructions

\section{Fin (Last Painting) de Stuart Davis : le mot comme clôture de l'œuvre peinte}

\section{Kamila Benayada}

\section{(2) OpenEdition}

\section{Journals}

Édition électronique

URL : http://journals.openedition.org/polysemes/5125

DOI : $10.4000 /$ polysemes. 5125

ISSN : 2496-4212

Éditeur

SAIT

\section{Référence électronique}

Kamila Benayada, «Fin (Last Painting) de Stuart Davis : le mot comme clôture de l'œuvre peinte », Polysèmes [En ligne], 21 | 2019, mis en ligne le 30 juin 2019, consulté le 11 juin 2019. URL : http:// journals.openedition.org/polysemes/5125; DOI : 10.4000/polysemes.5125

Ce document a été généré automatiquement le 11 juin 2019

Polysèmes 


\title{
Fin (Last Painting) de Stuart Davis : le mot comme clôture de l'œuvre peinte
}

\author{
Kamila Benayada
}

1 Le dernier tableau du peintre américain Stuart Davis, Fin (Last Painting), qui date de 1964, a pour titre l'un des mots qui composent l'œuvre, et sa traduction, voire une interprétation du mot français « fin ». Le mot ici explique autant qu'il décrit; il interprète et cette interprétation limite la lecture de l'œuvre. L'œuvre était encore sur le chevalet de Davis quand il est mort; il avait ajouté le mot « Fin » avant de mourir, alors que ce mot n'apparaît pas dans le croquis préliminaire que l'artiste avait fait. Le mot «fin » a donc pris une dimension particulière, celle de la fin de la vie, et de la fin de l'œuvre comme processus continu. Le mot, donc, est signifiant; et la barrière linguistique que l'artiste avait voulue a été écartée avec la traduction. L'artiste avait aussi introduit d'autres mots : « Front », ou encore les lettres O, W, et H. C'est pourtant le mot « Fin » qui l'a emporté. Le mot, donc, précise en même temps qu'il exclut l'interprétation. Il centre le regard, l'oriente, et empêche la libre lecture.

2 Pourtant, dans une lettre de 1952 au directeur du MoMA, Alfred Barr, Davis a expliqué : «I very often used words in my pictures, from the time I started art school, because words were often a part of the urban subject matter that I used » (Stuart Davis, lettre à Alfred Barr, directeur du MoMA, New York, 3 novembre 1952, reproduite dans Diane Kelder 100-102). En 1960, il a aussi écrit dans ses notes:

Words are a form, an analogue, of physical architecture/Words have meaning in reference to objective forms and acts/Words can refer to a precedent form or they can create one. (Stuart Davis Papers, 27 octobre 1960)

Les insertions verbales dans le domaine visuel sont donc à la fois sujet et objet, les mots entrent dans la toile comme d'autres formes perçues dans l'environnement. Certains mots réapparaissent, comme d'autres formes, d'une œuvre à une autre, contribuant ainsi à une continuité formelle et textuelle au sein de l'œuvre. Mais en même temps, ils restent des signifiants, des indications de sens ou d'interprétation. Le mot est cadre, il impose un sens, alors qu'il est employé contre le sens, comme forme supplémentaire, 
complémentaire dans la composition. L'artiste, en fait, hésite à donner aux mots un statut déterminé : ils sont signifiants, et simples signes, ils clôturent le sens et l'ouvrent en même temps, agissent comme limite, comme horizon, tout en franchissant les limites qu'ils construisent. À la frontière entre sens et non-sens, le mot est indétermination, instabilité, in-finitude. Il résiste ainsi à la clôture des lectures interprétatives.

\section{Le mot dans l'œuvre : le concept utopique de color- space}

En 1954, Davis explique :

I have used words in many of my pictures, and in all cases they are used in the same sense and feeling as the nonword shapes. [...]. Words in an infinite variety of visual forms are an important part of the subject matter of our daily experience. I can think of no good reason to exclude them where they present themselves spontaneously to my attention. I accept or reject their shape in the same way as any other shape. Whatever specific context of meaning they have is changed into their context of meaning as a part of the total meaning of the picture. [...] As to the title, I select words in much the same way [...] when they seem appropriate in sound, not for any descriptive reason. (Stuart Davis, memo à Henry Clifford, directeur du Philadelphia Museum of Art, 17 avril 1954, reproduit dans Kelder 103-104)

Les mots, donc, n'auraient aucune fonction signifiante. Le mot est déclassé, l'artiste l'extrait des réseaux de sens préexistants. Il est transformé en simple forme, il entre dans un réseau de signes qui lui donne un sens nouveau.

Les mots comme simples éléments du paysage apparaissent dès le début de la carrière de Davis. Par exemple, dans Landscape (1922), l'artiste intègre la signalisation routière aperçue dans le paysage ( «curve », "go slow»), et les mots ont le même statut que les différentes parties du paysage. On retrouve les mêmes éléments dans une toile de 1957, Composition Concrete, mais Davis met au point sur le centre de la toile, et les mots disparaissent ; il ne reste que les lettres $C$ et $\mathrm{U}$ de «Curve ». Ce recentrage fragmente la toile initiale, et les éléments reconnaissables (formes et mots) sont neutralisés, réduits à des éléments structurels. Davis fait la même chose dans Blips and Ifs (1963-64), qui est une version agrandie du centre de Standard Brand (1961). Là encore, les mots qui étaient entiers dans Standard Brand sont fragmentés, la continuité initiale est annulée, ce qui était « complet » dans Standard Brand ne l'est plus dans Blips and Ifs.

5 L'emploi de mots étrangers, en particulier dans les titres, contribue à ce processus de destruction du contenu signifiant des mots (Semé (1953), Pochade (1958), Stele (1956), Allée (1955), Bois (1960), Château (1960), ou encore Composition Concrete). Ils deviennent alors étranges, et leur altérité rend impossible leur appréhension en tant que mots. Ils échappent à la matrice culturelle dans laquelle le lecteur/spectateur évolue, matrice qui influence la compréhension. Les mots étrangers, en ce qu'ils sont en dehors de cette matrice, ont une liberté dont Davis se sert : il peut alors les réduire à leur simple forme, les intégrer dans l'œuvre non plus comme mot, mais comme surface plane à colorer. Ils participent à la seule unité formelle de l'œuvre, et pas à une unité de sens. Davis veut employer les mots (comme les formes) sans leur fonction de représentation. Les deux (mots et formes) coexistent sur un même plan visuel, ils sont des signes réversibles, au sein d'une image continue. Mais cette continuité est formelle; elle fonctionne à l'encontre et en dépit de la discontinuité de la fusion mot/image. Davis a expliqué dans 
un entretien : «Physically, words are also shapes » (Kuh 52). Le mot, donc, est un signe non signifiant, il est une forme comme une autre.

Davis écrit en 1962, «Letters LOCK Scale / Letters LOCK Color... » (Stuart Davis Papers, 5 février 1962). Le mot est une limite, une entrave à la couleur, et il délimite l'échelle de la composition; il entoure et définit des espaces dans la toile. Davis traite les mots comme des espaces clos, il leur attribue des couleurs, et ces couleurs ont une fonction dans la composition: la forme du mot est alors une forme colorée («color-shape») qui est juxtaposée à d'autres formes colorées. La juxtaposition crée des différences, et c'est dans cette différence que se trouve la fonction des formes. Leur sens est à la fois syntaxique, et différentiel. Dans Premiere (1957), Davis part d'emballages de produits de consommation et ne retient que certains mots qu'il place dans des plans de couleur. Son objectif était de créer des "intervalles optiques" (optical intervals), où la couleur indiquerait des différences, des ruptures dans les positions des formes (Sims 296). Cette juxtaposition crée une contradiction simultanée :

[...] the presence of two opposing space systems [...that] establish a specific planal [ sic] relationship. [...] The dialectical elements, whose contradictory simultaneous experience creates the aesthetic form, are as follows: length, area, color-tone. (Sims 60)

7 En gardant l'idée saussurienne du sens créé par la différence, cet emploi des mots, qui est au départ une transgression du visuel, est une transgression du langage. Le mot est pris dans sa matérialité, voire son opacité, puisqu'il n'a plus de sens. Il est hors contexte, hors code. Alors, les mots «Fin », " Front », et " Owh», dans Fin (Last Painting), auraient une valeur formelle équivalente : «Fin » et «Front » sont composés de lettres noires, « Owh » de deux lettres noires et une verte au milieu; ces mots sont dans des espaces de couleurs différentes, et ces espaces sont eux-mêmes délimités par des bandes de papier-cache adhésif. Ils divisent l'espace comme les bandes adhésives noires et beige, et comme les autres plans de couleur. Ils entravent le mouvement de la pensée, l'interprétation qui passerait par la lecture ; le mot est suspension aporique du mouvement.

8 En fait, le sens viendrait après coup, de l'intégration des mots comme formes dans la composition. C'est l'œuvre qui donne son sens aux formes qui la composent et non pas l'inverse. «The Alphabet Syntax and Language of Color-Space Method becomes the Object [...] as they take the place of Subject as Given » (Stuart Davis Papers, 6 juin 1960). Ce n'est ni le « sujet ", la forme trouvée dans la nature, ni le mot signifiant qui crée la composition, c'est la structure de l'espace en zone de couleurs (color-space) : ce qui est donné au préalable (« subject as given»), qu'il s'agisse des formes trouvées dans l'environnement ou des mots, n'a de sens que dans la mesure où ils entrent dans l'espace couleur.

Les formes organisées dans la toile obéissent à leur propre grammaire et elles sont un alphabet que l'artiste travaille. En ôtant le mot des réseaux de sens que lui donne la culture, Davis a l'ambition utopique de leur en donner un nouveau, qui ne serait pas contaminé par le langage. C'est son langage pictural à lui qui façonne le sens des formes. Les mots ont une nouvelle identité, une nouvelle fonction, qu'ils trouvent au-delà des limites du langage. Leur emploi équivoque les détourne de leur fonction signifiante, de leur fin annoncée: ils ont une autre destination que celle du sens; ils dépassent le langage et s'en libèrent. Le mot serait alors asocial : il perdrait son rôle communicant, en dépassant l'horizon du sens. Sa présence dans la toile et dans l'œuvre du peintre est aussi une absence de sens. Mais les mots enferment autant le sens que les couleurs : ils donnent une tonalité à la toile, une orientation à la lecture. Le projet formaliste de Davis est 
essentiellement utopique : l'objectivité visuelle à laquelle il aspire est impossible, les mots ne peuvent échapper au langage, à leur rôle de signifiant.

\section{Le mot comme clôture}

10 La toile Fin (Last Painting) est la dernière d'une série ayant pour point de départ une photographie trouvée par l'artiste dans le New York Times. Le squelette reste le même dans cette série, mais Davis ajoute ou enlève certains éléments, change les couleurs, et dans cette ultime toile, il rajoute les mots « Fin », « Front », et " Owh ». Si l'on compare la toile peinte à l'étude que Davis en avait faite, on voit que le mot «Fin» n'était pas prévu initialement. Davis a écrit, en haut du croquis, « in-out field» et « sans foin » (sans fin ?). Ces indications sont pour l'artiste, elles ne doivent pas figurer dans la version finale. On voit cependant que Davis s'intéressait au champ (la toile ou le plan, l'aplat comme champ) comme lieu d'entrée et de sortie, comme lieu de migration infinie.

11 «Front» et "Owh» sont des mots qui apparaissent ailleurs dans l'œuvre de Davis. Ils contribuent donc à une continuité de l'œuvre. Ils sortent d'autres œuvres pour entrer dans celle-ci. Mais en même temps, ils constituent un vocabulaire spécifique au peintre, un lexique de formes qui pourtant, déjà, font sens. «Front », par exemple, figure sur la couverture de la revue du syndicat des artistes Art Front, que Davis avait réalisée en 1935. "Owh» apparaît dans le titre d'une toile de 1951 (Owh! in San Pao), mais pas dans la peinture elle-même. «Pao » figurait dans Emblem (1963), l'une des toiles de la série. Les mots migrent du titre dans la toile. Il y a aussi migration des indications personnelles vers l'intérieur de la toile («Fin», et "Front» qui figure aussi en haut du croquis préliminaire). Davis crée ainsi un réseau continu de toiles liées non seulement par la forme ou le croquis originel, mais aussi par les mots. Pourtant, les mots restent opaques, obscurs, ils ne signifient pas, ils sont encore hors langage, silencieux.

Néanmoins, les mots ont aussi valeur de signifiants : dès 1921, Davis donnait aux mots une fonction spécifique, celle de sous-titre ou de légende : «Words will have a place [in my art] also (just as in movies captions are necessary)» (Stuart Davis Notebook, Sims 153). Le visuel seul semble ne pas lui suffire. Plus tard, en 1952, il confirme que le sens des mots joue un rôle dans l'œuvre :

One could say that any [...] word that filled that area would be as good as the one used, but this would not be the case because, while there could be any number of words which occupied the same space and performed the same color-space structural function, their specific meaning would have an importance that would divert the attention. (Kelder 102)

13 Le choix des mots est donc calculé. Dans la série qui aboutit à Fin (Last Painting) et que Davis commence en 1960, les titres des premières toiles sont une référence directe à la photographie du château de Carcassonne trouvée dans le New York Times, une description qui oriente la lecture: Bois, Château, Terrace. L'inclusion des mots "Front ", "Owh", et «Fin» dans le dernier tableau est donc plus qu'une exploration des relations formelles entre les formes que composent le mot et les formes qui l'entourent.

Entre 1960 et 1964, il peint d'autres toiles dont la palette réduite est la même que pour la série. Le mot "Any " migre d'une toile à l'autre; on le retrouve dans Standard Brand (1961-62), ou dans Thermos (1962), où Davis écrit en bas à gauche : « Any as Given ». Davis explique dans ses notes que le mot "Any » est un rappel du manque d'importance du sujet ; mais le mot est placé dans la toile pour sa forme autant que pour son sens. Le mot a 
un double rôle structurel : «Any » ouvre la possibilité de sujets pour l'artiste, car tout sujet est bon, mais en même temps, le mot est récurrent, et toujours en lettres cursives, et il interrompt la géométrie de Standard Brand.

Paradoxalement, donc, les mots introduisent une instabilité dans la toile; les mots rompent les lignes que créent les plans de couleur, ils interrompent les aplats. Les insertions verbales fragmentent non seulement l'espace-couleur (color-space) mais elles fragmentent la lecture, nous faisant passer du visuel au textuel. La continuité formelle des toiles est rompue par l'arrivée incongrue d'un autre système référentiel. Les mots troublent la surface plane; les typographies variables (lettres cursives pour la signature ou pour certains mots, variations de la taille des lettres) se heurtent à la fixité apparente des autres formes plates; alors que les autres formes purement visuelles ont une certaine stabilité, d'une toile à l'autre, les mots, viennent s'y ajouter, comme collés sur un fond auquel ils n'appartiennent pas vraiment. Le champ de migration est déplacé; aux espaces-couleurs se superposent l'espace linguistique et l'espace conceptuel de l'artiste. Superpositions, allées et venues, Davis peint dans un univers instable, perméable, in-fini.

Alors qu'en 1961, Davis annonce dans la toile Standard Brand «Complet»; en 1962, Davis peint Unfinished Business, puis en 1963, la version revue de Standard Brand qu'est Blips and Ifs coupe le mot « Complet ». Les mots, donc, nous envoient dans une direction, même si l'artiste change de direction plus tard. Alors que la série sur Carcassonne migre peu à peu vers la version finale, l'artiste se pose encore des questions sur ce qui constitue une œuvre finie (" complet », « unfinished»). Si la toile est un champ de migration (« in-out field »), la migration se fait d'une toile à l'autre, et on « lit » le cheminement de la pensée du peintre, ses obsessions, ses insatisfactions, dans les mots qu'il intègre à la composition. Plus qu'une forme intégrée à d'autres formes, le mot donne forme à l'œuvre comme processus ou comme projet.

\section{L'horizon ouvert : le mot/l'œuvre comme indétermination, errance}

On l'a vu, Davis lui-même nous égare dans ses écrits: ses indications contradictoires et obscures n'aident pas l'interprétation, au contraire, ses mots font parfois obstacle à la lecture de son œuvre. Ainsi, le mot n'est pas simple forme, il n'est pas non plus simple indication de lecture, orientation; le mot est instable, il est multiple dans son unité. Et cette instabilité ouvre l'œuvre au lieu de l'encadrer.

Davis joue sur l'arbitraire, la polysémie, en ne nous donnant que des mots, seuls, sans les encadrer dans des phrases, en ne les encadrant que par des plans de couleurs. Le mot n'existe pas dans ses rapports à d'autres mots, il est hors langage; il existe seul, autonome, ou en relation avec la composition où il est intégré, ou encore en relation avec le même mot trouvé ailleurs dans l'œuvre de Davis. Sorti des réseaux codés qui imposent un sens, le mot est libre de signifier tout ce qu'il peut. Et cette polysémie multiplie les sens possibles de la toile où s'inscrit le mot. La clôture du non-sens, du non-langage est aussi, paradoxalement, ouverture. En transcendant le sens, Davis ouvre le mot à des possibilités infinies. En reprenant le même mot dans un contexte visuel différent, il explore les multiples possibilités formelles et significatives du mot.

Le mot, comme l'œuvre de Davis, est ainsi processus, à la fois continu et discontinu : chaque mot, comme chaque œuvre est un moment, une station, qui met en marche 
l'œuvre dans sa totalité et sa continuité. La toile est une étape, où se sédimentent d'autres œuvres; le mouvement "in-out» se poursuit d'une toile à l'autre, mots, formes et couleurs migrent. Davis établit ainsi une durée, dans la continuité du processus, plutôt que dans chaque toile individuelle. L'œuvre est retardée, la toile est toujours « unfinished business »; ce qui est « complet » dans une toile (Standard Brand) doit être revu dans la suivante (Blips and Ifs), détruit, pour que le processus reparte. Et le titre Blips and Ifs le confirme : chaque toile est à la fois un «blip » et un « if », une déviation et une possibilité.

En 1963, Davis peint Contranuities, reprenant en le transformant, le mot « Continuity » qui apparait dans Visa (1951) (le mot est coupé : il est composé de "Continu »- et «-ity »). On a expliqué que "Contranuities » voulait en fait dire « contra-new-it-is » (against the new), donc Davis s'oppose à la nouveauté, et reprend ses toiles passées pour créer de nouvelles toiles. La continuité est le déplacement du passé, le mouvement du passé d'une toile à l'autre, « in-out ». Le passé est « unfinished business », qui doit être repris, revu ; la toile n'est jamais fermée, elle reste ouverte.

L'œuvre est errance. Davis passe d'une photographie du château de Carcassonne à un croquis où il retient certaines formes de la photographie, il divise la toile horizontalement et verticalement, et il rajoute le mot «check » à cheval entre la partie gauche et la partie droite. Ce croquis, qui sert de base à la gouache Bois, nous montre aussi comment cette division de la toile permet à l'artiste de passer de Bois à Closed Circuit (là encore, le circuit fermé des mêmes formes qui reviennent, "Contranuities ", s'oppose à « unfinished business » et fait référence au circuit électrique qui permet la circulation du courant), et ensuite de passer de Closed Circuit à Terrace, par exemple, et le travail du peintre se poursuit jusqu'à la dernière toile. Le mot «Check » renvoie aux vérifications multiples que fait l'artiste des possibilités de composition qu'offre la photographie initiale. Il s'agit de faire échec au sens fixe, à l'horizon impossible, à l'aporie. La toile et l'œuvre sont des champs d'entrée et de sortie.

Ce que Davis introduit dans ses œuvres, et dans son œuvre prise dans sa globalité, c'est l'indéterminé, la différance, le mouvement. Son parcours est une errance, un passage perpétuel, et il s'oppose, dans ses articles, à l'interprétation qui limite le sens, impose un horizon. Le silence, la polysémie, les incohérences, le non-sens, les discontinuités sont toutes des démarches contre la stabilité de l'œuvre. Le mot est alors à la frontière entre sens et indétermination : il indique, tout en limitant la compréhension. Il ouvre l'horizon, tout en le fermant. Simple forme, il est sans signification autre que formelle. En tant que forme, il réapparaît dans différentes œuvres, parfois dans le titre, parfois dans la toile. Élément interne, il ouvre la toile en l'intégrant dans une continuité, une démarche à la fois esthétique et intellectuelle, car le mot, finalement, ne peut être qu'une simple forme, ni avoir un sens unique. Le mot «Fin » apparaît une seule fois dans ses toiles, dans la dernière. Et cette dernière peinture est le dernier point d'entrée de son œuvre. Il finit un travail sur l'in-fini, encadre la toile (comme le cadre noir peint par Davis, et les bandes adhésives), lui donne un sens déterminé, y met un point final. C'est moins un aboutissement qu'un point de rupture, où l'artiste se rend compte que son travail sera interrompu. Le mot «Fin», en fermant l'horizon, contredit le principe même de l'esthétique de Davis qui est l'in-finitude. 


\section{BIBLIOGRAPHIE}

\section{Fuvres de Stuart Davis citées}

(Toutes les œuvres citées sauf Bois, Château, Terrace, Allée, Emblem, Closed Circuit sont sur www.artnet.com)

Curve Go Slow, 1922, huile sur toile, 38,74x29,21 cm, Yale University Art Gallery, New Haven, Connecticut.

Owh! In San Pao, 1951, huile sur toile, 132,72x106,04 cm, Whitney Museum of American Art, New York.

Semé, 1953, huile sur toile, 132,08x101,6 cm, The Metropolitan Museum of Art, New York.

Allée, 1955, huile sur toile, 243,8x1005,8 cm, Drake University, Olmsted Center, Des Moines, Iowa. Stele, 1956, huile sur toile, 132,72x101,93 cm, Milwaukee Art Museum, Milwaukee, Wisconsin. Composition Concrete, 1957, huile sur toile, 515 x 244,3 cm, Carnegie Museum of Art, Pittsburg, Pennsylvanie.

Première, 1957, huile sur toile, 147,3X127 cm, Los Angeles County Museum of Art, Los Angeles, Californie.

Pochade, 1956-58, huile sur toile, 132,08x152,4 cm, Museo Thyssen-Bornemisza, Madrid, Espagne. Bois, 1960, gouache sur papier, repr. in Lowery Stokes Sims (ed.), Stuart Davis, American Painter, cat. exp., The Metropolitan Museum of Art, New York: Harry N. Abrams, 1991, 90.

Château, 1960, gouache sur papier, repr. in Lowery Stokes Sims, Stuart Davis, American Painter, cat. exp., The Metropolitan Museum Of Art, New York: Harry N. Abrams, 1991, 90.

Terrace, 1960, Huile sur panneau, repr. in Lowery Stokes Sims, Stuart Davis, American Painter, cat. exp., The Metropolitan Museum Of Art, New York: Harry N. Abrams, 1991, 91.

Standard Brand, 1961, huile sur toile, 152,4x116,84 cm, The Detroit Institute of Arts, Detroit, Michigan.

Tournos, 1954, huile sur toile, 91,1x71,1 cm, repr. in Lowery Stokes Sims, Stuart Davis, American Painter, cat. exp., The Metropolitan Museum Of Art, New York: Harry N. Abrams, 1991, 244.

Closed Circuit, 1962, caséine sur papier, repr. in Lowery Stokes Sims, Stuart Davis, American Painter, cat. exp., The Metropolitan Museum Of Art, New York: Harry N. Abrams, 1991, 91.

Unfinished Business, 1962, huile sur toile, $91,44 \times 114,3 \mathrm{~cm}$, copyright The Estate of Stuart Davis, VAGA, NY, NY.

Contranuities, 1963, huile sur toile, 172,72x $127 \mathrm{~cm}$, copyright The Estate of Stuart Davis, VAGA NY, NY.

Emblem, 1963, caséine sur papier, repr. in Sims, Stuart Davis, American Painter, cat. exp., The Metropolitan Museum Of Art, New York: Harry N. Abrams, 1991, 92.

Blips and Ifs, 1963-64, huile sur toile, The Amon Carter Museum, Fort Worth, Texas. 
Fin (Last Painting), 1964, caséine et ruban sur toile, 136,86x100,96 cm, copyright, The Estate of Stuart Davis, VAGA NY, NY.

\section{Ouvrages cités}

Kelder, Diane (ed.). Stuart Davis: Art and Theory 1920-31. New York: Pierpont Morgan Library, 2002.

Kuh, Katharine. The Artist's Voice: Talks with Seventeen Artists. New York: Harper and Row, 1960.

Davis, Stuart. Stuart Davis Papers. Harvard University Art Museums, Fogg Art Museum, Cambridge, Mass.

Sims, Lowery Stokes (ed.). Stuart Davis, American Painter. Cat. Exp. The Metropolitan Museum of Art, New York: Harry N. Abrams, 1991.

\section{RÉSUMÉS}

Cette analyse du dernier tableau du peintre américain Stuart Davis met en relation cette œuvre avec d'autres peintures de Davis, faisant apparaître la structure de l'œuvre comme superposition d'espaces-couleur, d'espaces linguistiques et d'espaces conceptuels. La toile, comme les éléments qui la composent, est un champ de migration et de transgression (visuelle et linguistique). Mais le projet d'objectivité de Davis, qui constitue pour lui la finalité de l'art, est utopique, les mots ne pouvant échapper à leur rôle de signifiant.

Through bringing together this and other paintings by this American artist, this analysis of Stuart Davis's last painting points to the structure of his paintings as multiple spaces, "colorspace", linguistic space, and conceptual space. The painting, and its structural elements, is an "in-out field", a field of migration and transgression, both visual and verbal. But Davis's project to create objective art, objectivity being the end of art, its finality, appears as utopian in view of the inescapable significance of words.

\section{INDEX}

oeuvrecitee Fin (Last Painting)

Mots-clés : signifiant, signifié, migration, transgression, langage, processus, rupture, utopie

Keywords : signifier, signified, migration, transgression, language, process, rupture, utopia

\section{AUTEURS}

\section{KAMILA BENAYADA}

Kamila Benayada est maître de conférences en études américaines à l'université d'Orléans (PoLEN). Sa spécialité est la peinture des États-Unis, notamment Stuart Davis, Thomas Hart Benton, le régionalisme, et le premier modernisme. Elle a publié dans LISA, RFEA, Interfaces, IdEA, Polysèmes, Textes et Contextes, Transatlantica, et dans Essais sur l'art (dir. Mathilde Arrivé), Histoire et analyse des images dans la culture américaine (dir. François Brunet). 